\title{
O Benefício da Terapia Cognitivo- -Comportamental na Tontura Persistente Postural-Paroxística
}

\section{The Benefits of Cognitive Behavior Therapy in Persistent Postural-Paroxysmal Dizziness}

José Neves ${ }^{\# 1}$, Sara Araújo Machado\#2, José Luís Castro², Pedro Barreira³, Raquel Sanches ${ }^{4}$

\section{RESUMO}

INTRODUÇÃO: A tontura persistente postural-paroxística é uma entidade recentemente reconhecida, que engloba cerca de 10\% dos doentes com queixas de tontura. O seu tratamento passa pela terapêutica farmacológica com inibidores seletivos da recaptação da serotonina, associada ou não a um programa de reabilitação vestibular. Tendo em conta a difícil gestão destes doentes e resistência ao tratamento convencional, coloca-se a hipótese do uso da terapia cognitivo-comportamental no tratamento desta patologia.

O objetivo deste trabalho é determinar se existe benefício da terapia cognitivo-comportamental no tratamento da tontura persistente postural-paroxística.

METODOLOGIA: Foi realizada uma pesquisa usando as palavras-chave "persistent postural perceptual dizziness", "cognitive behavioural therapy", "phobic postural vertigo", nas seguintes bases de dados: National Guideline Clearinghouse, Canadian Medical Association Practice Guidelines InfoBase, DARE - Database of abstracts of reviews of effectiveness, Evidence-Based Medicine e PubMed. Foram ainda incluídos artigos referenciados na bibliografia da pesquisa inicial.

RESULTADOS: Foram selecionados três artigos nas bases de dados. Foram incluídos dois artigos adicionais referenciados na bibliografia inicial. No total foram incluídos cinco artigos: dois estudos caso-controlo e três revisões de literatura.

DISCUSSÃO: É consistente o benefício da terapia cognitivo-comportamental como parte de uma abordagem multidisciplinar. Foram reportadas, nestes doentes, melhoria das queixas de tontura, ansiedade e depressão. Verificou-se ainda melhoria do controlo postural e diminuição da dose de medicação necessária. Os efeitos da terapia cognitivo-comportamental a longo prazo são ainda pouco consistentes.

\#Os autores contribuíram de igual forma para a elaboração do presente trabalho/ \#Joint first authors

1. Interno de Formação Específica em Medicina Geral e Familiar, USF Monte Castro, ACES Gondomar, Gondomar, Portugal. 2. Interno de Formação Específica em Medicina Geral e Familiar, USF Samora Correia, ACES Estuário do Tejo, Samora Correia, Portugal. 3. Interno de Formação Específica em Medicina Geral e Familiar, USF AIcais, ACES Cascais, Cascais, Portugal. 4. Interno de Formação Específica em Medicina Geral e Familiar, USF S. Julião, ACES Oeiras e Lisboa Ocidental, Oeiras, Portugal.

• Autor (es) (ou seu (s) empregador (es)) 2019. Reutilização permitida de acordo com CC BY-NC. Nenhuma reutilização comercial. ${ }^{\bullet}$ Author(s) (or their employer(s)) 2019. Re-use permitted under CC BY-NC. No commercial re-use. 
CONCLUSÃO: A terapia cognitivo-comportamental parece ser uma opção terapêutica válida, em associação com outras medidas. Destaca-se a escassez de evidência científica nesta área, e a necessidade de estudos adicionais.

PALAVRAS-CHAVE: Terapia Cognitivo-Comportamental; Tontura/tratamento; Vertigem Posicional Paroxística Benigna/tratamento

\section{ABSTRACT}

INTRODUCTION: Persistent postural-paroxysmal dizziness is a recently recognized entity, affecting about 10\% of the patients complaining with dizziness. Its treatment involves pharmacological therapy with selective serotonin reuptake inhibitors, associated or not with a vestibular rehabilitation program. Given the difficult management of these patients and resistance to conventional treatment, cognitive behavioral therapy appears as a treatment possibility for this condition.

The objective of this study is to determine if there is benefit in using cognitive behavioral therapy in the treatment of persistent postural-paroxysmal dizziness.

METHODOLOGY: A search was performed using the keywords "persistent postural perceptual dizziness", "cognitive behavioral therapy", "phobic postural vertigo" in the following databases: National Guideline Clearinghouse, Canadian Medical Association Practice Guidelines InfoBase, DARE - Database of abstracts of reviews of effectiveness, Evidence-Based Medicine and PubMed. Other articles mentioned in the initial research were also included.

RESULTS: Three articles were identified in the databases. Two additional articles mentioned in the initial research were included. In total five articles were included: two case-control studies and three literature reviews.

DISCUSSION: The benefit of cognitive behavioral therapy as part of a multidisciplinary approach is consensual. Improvements in dizziness, anxiety and depression have been reported in these patients. There was also improvement in postural control and decrease in the medication dosages required. The long-term effects of cognitive behavioral therapy are still inconsistent. CONCLUSION: Cognitive behavioral therapy seems to be a valid therapeutic option in association with other measures. We stress the lack of scientific evidence in this area and the need for further studies.

KEYWORDS: Benign Paroxysmal Positional Vertigo/therapy; Cognitive Behavioral Therapy; Dizziness/therapy

\section{INTRODUÇÃO}

A tontura persistente postural-paroxística (TPPP) é uma entidade recentemente reconhecida e listada na $11^{a}$ revisão da Classificação Internacional de Doenças (CID-11). Previamente com outras designações, como vertigem postural fóbica (VPF), refere-se a uma doença de caráter crónico, caraterizada por tontura persistente não-vertiginosa, sensação de instabilidade, ou ambos, com duração de pelo menos 3 meses. ${ }^{1,2}$

A sua fisiopatologia permanece desconhecida. No entanto, compreende-se que a vertigem posicional paroxística benigna (VPPP) constitui uma disfunção do sistema de equilíbrio e processamento vestibular, sem nenhuma lesão de órgão específica identificada até então. ${ }^{2,3}$

Trata-se de uma patologia frequente na prática clínica, constituindo cerca de $10 \%$ dos doentes com queixas de vertigem/tontura, mais comum em mulheres dos 40-60 anos. 3,4

O tratamento da TPPP passa por medidas farmacológicas e não-farmacológicas. Os inibidores seletivos da recaptação da serotonina (SSRI's) são os fármacos de eleição no tratamento desta patologia, no entanto, cerca de 1/3 dos doentes não sentem melhoria sintomática ou não toleram os seus efeitos secundários. ${ }^{1-3}$ Para além das medidas farmacológicas, a reabilitação vestibular (RV) é uma terapia bem estudada e usada no tratamento desta patologia, com resultados significativos nos doentes com componente vestibular predominante. No entanto, este trata-se de um recurso de difícil acesso, apenas disponível em centros especializados..$^{1,2}$

Sendo que se trata de uma doença crónica, de difícil gestão e com poucas armas terapêuticas, consideramos relevante explorar outras abordagens que ajudem no seu controlo. Mais recentemente foi colocada a hipótese do uso da terapia cognitivo-comportamental (TCC) no tratamento destes doentes.

O objetivo deste trabalho é determinar se existe benefício no uso da TCC no tratamento da TPPP.

\section{MÉTODOS}

Foi realizada uma pesquisa usando as palavras-chave "persistent postural perceptual dizziness", "cognitive behavioural therapy", "phobic postural vertigo", usando 
as seguintes bases de dados: National Guideline Clearinghouse, Canadian Medical Association Practice Guidelines InfoBase, DARE - Database of abstracts of reviews of effectiveness, Evidence-Based Medicine e PubMed. Foram ainda incluídos artigos referenciados na bibliografia da pesquisa inicial considerados relevantes.

\section{RESULTADOS}

Foram identificados quatro artigos relevantes nas bases de dados utilizadas, tendo sido eliminado um artigo após leitura por não ser relevante para o tema. Foram ainda incluídos dois artigos adicionais referenciados nos artigos da pesquisa inicial (Fig. 1). No total foram incluídos cinco artigos para análise, dois estudos caso-controlo e três revisões de literatura. Os resultados foram resumidos nas Tabelas 1 e 2.

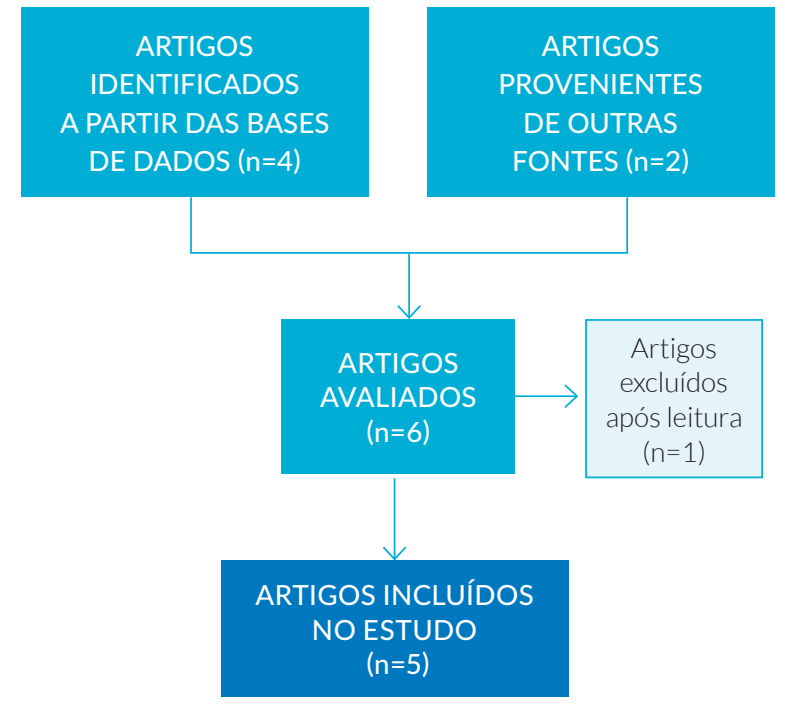

FIGURA 1. Fluxograma de seleção dos artigos.

TABELA 1. Sumário dos estudos de revisão. Graus de recomendação e Níveis de evidência estabelecidos de acordo com o método SORT ("Strength of Recomendation Taxonomy").

\begin{tabular}{|c|c|c|c|c|c|c|c|}
\hline Autor & Ano & Tipo & Objetivos & Métodos & Conclusões & GR & NE \\
\hline Popkirov et al ${ }^{1}$ & 2018 & $\begin{array}{l}\text { Revisão não } \\
\text { sistemática }\end{array}$ & $\begin{array}{l}\text { Revisão das } \\
\text { diferentes } \\
\text { estratégias } \\
\text { terapêuticas para } \\
\text { a TPPP e fornecer } \\
\text { recomendações } \\
\text { práticas para } \\
\text { uma abordagem } \\
\text { multidisciplinar. }\end{array}$ & & $\begin{array}{l}\text { A TCC parece ser uma intervenção } \\
\text { útil no tratamento desta patologia, } \\
\text { apesar dos seus efeitos a longo } \\
\text { prazo ainda estarem por estabelecer. } \\
\text { Parece mais eficiente quando } \\
\text { em combinação com técnicas de } \\
\text { reabilitação vestibular e/ou técnicas } \\
\text { de relaxamento. }\end{array}$ & B & 2 \\
\hline $\begin{array}{l}\text { Trinidade A, } \\
\text { Goebel JA. }\end{array}$ & 2018 & $\begin{array}{l}\text { Revisão } \\
\text { sistemática }\end{array}$ & $\begin{array}{l}\text { Revisão bibliográfica } \\
\text { da informação } \\
\text { disponível acerca } \\
\text { da TPPP e } \\
\text { apresentar critérios } \\
\text { diagnósticos, assim } \\
\text { como estratégias } \\
\text { e abordagens } \\
\text { terapêuticas. }\end{array}$ & $\begin{array}{l}\text { Foram usadas as palavras-chave } \\
\text { "persistent postural-perceptual } \\
\text { dizziness" e "PPPD" nas } \\
\text { seguintes plataformas: CINAHL, } \\
\text { Embase, PubMed, Medline, } \\
\text { PsycINFO, PubMed, Google } \\
\text { Scholar. } \\
\text { Foram selecionados } 15 \text { artigos: } \\
1 \text { caso-controlo randomizado; } \\
4 \text { revisões não sistemáticas; } \\
7 \text { estudos caso-controlo; } \\
1 \text { revisão retrospetiva; } \\
1 \text { caso clínico e } 1 \text { consenso. }\end{array}$ & $\begin{array}{l}\text { O tratamento da TPPP deve assentar } \\
\text { numa abordagem multidisciplinar } \\
\text { com recurso à educação do doente, } \\
\text { reabilitação vestibular, medicação } \\
\text { (SSRIs e inibidores seletivos da } \\
\text { recaptação de noradrenalina) e TCC. }\end{array}$ & B & 2 \\
\hline $\begin{array}{l}\text { Whalley MG, } \\
\text { Cane DA. }\end{array}$ & 2017 & $\begin{array}{l}\text { Revisão não } \\
\text { sistemática }\end{array}$ & $\begin{array}{l}\text { Revisão acerca } \\
\text { da evolução do } \\
\text { diagnóstico e } \\
\text { tratamento da TPPP } \\
\text { e apresentação de } \\
\text { uma abordagem } \\
\text { centrada na TCC. }\end{array}$ & & $\begin{array}{l}\text { Os autores consideram que na } \\
\text { TPPP, os doentes que apresentam } \\
\text { comportamentos de evasão e } \\
\text { preventivos, assim como uma maior } \\
\text { suscetibilidade a desenvolverem } \\
\text { sintomatologia psicossomática } \\
\text { determinada pelo medo da tontura, } \\
\text { apresentam um certo grau de } \\
\text { disfunção cognitivo-comportamental. } \\
\text { Nestes doentes, a vertigem tende a } \\
\text { adquirir um caráter persistente. } \\
\text { Os autores consideram, desta forma, } \\
\text { que a TCC deveria estar incorporada } \\
\text { em todas as metodologias de } \\
\text { tratamento da TPPP. }\end{array}$ & $C$ & 3 \\
\hline
\end{tabular}


TABELA 2. Sumário dos estudos de caso-controlo; Graus de recomendação e Níveis de evidência estabelecidos de acordo com o método SORT ("Strength of Recomendation Taxonomy").

\begin{tabular}{|c|c|c|c|c|c|c|}
\hline Autor & $\begin{array}{l}\text { Ano } \\
\text { País }\end{array}$ & Amostra & Objetivos & Metodologia/Resultados/Limitações & GR & NE \\
\hline $\begin{array}{l}\text { Schmid } \\
\text { et al }\end{array}$ & $\begin{array}{l}2018 \\
\text { Suíça }\end{array}$ & $\begin{array}{l}\mathrm{N}=32 \\
\text { Idade média: } \\
\text { - Casos: } \\
\text { 60,6 } \pm 8,3 \text { anos } \\
\text { - Controlos: } \\
\text { 44,8 } \pm 12,1 \text { anos } \\
\text { Género (M:F): } \\
\text { 23:9 }\end{array}$ & $\begin{array}{l}\text { Determinar } \\
\text { a eficácia de } \\
\text { um programa } \\
\text { terapêutico } \\
\text { combinado com } \\
\text { TCC, reabilitação } \\
\text { vestibular e } \\
\text { psico-educação em } \\
\text { doentes com e sem } \\
\text { patologia vestibular. }\end{array}$ & $\begin{array}{l}\text { Metodologia: O grupo de } 32 \text { pacientes foi dividido em dois subgrupos. } \\
\text { O grupo de casos consistiu em } 16 \text { doentes com tontura com patologia } \\
\text { vestibular e o grupo de controlo consistiu em doentes com tontura sem } \\
\text { patologia vestibular. Todos os pacientes foram submetidos a questionários } \\
\text { (BSI e DHI) pré e pós-tratamento. } \\
\text { Ambos os grupos foram submetidos ao mesmo programa terapêtico. } \\
\text { Resultados: } \text { O equilíbrio postural melhorou significativamente no grupo } \\
\text { de pacientes com patologia vestibular (p=0,003) mas tal não se verificou } \\
\text { no grupo sem patologia vestibular. Os índices dos questionários DHI e } \\
\text { BSI melhoraram significativamente no grupo sem patologia vestibular, } \\
\text { no entanto, não houve qualquer impacto nestes índices no grupo de } \\
\text { pacientes com patologia vestibular. Os índices de ansiedade fóbica } \\
\text { melhoraram substancialmente para ambos os grupos, com maior impacto } \\
\text { no grupo sem patologia vestibular. } \\
\text { A combinação de TCC, reabilitação vestibular e psico-educação provou } \\
\text { melhorar os índices psicológicos no grupo sem patologia vestibular, no } \\
\text { entanto, não substancialmente no grupo com patologia vestibular, apesar } \\
\text { da melhoria marcada no equilíbrio postural. } \\
\text { Limitações: Diferença substancial de idade média entre os grupos; } \\
\text { follow-up de apenas } 2 \text { meses. }\end{array}$ & B & 2 \\
\hline Yuet al ${ }^{3}$ & $\begin{array}{l}2018 \\
\text { China }\end{array}$ & 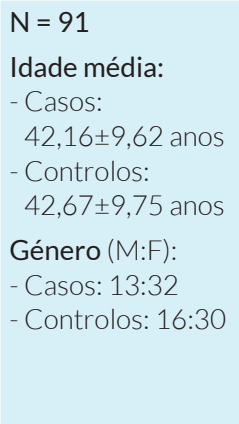 & $\begin{array}{l}\text { O papel da terapia } \\
\text { cognitivo- } \\
\text {-comportamental } \\
\text { como terapia } \\
\text { potenciadora } \\
\text { para pacientes } \\
\text { diagnosticados com } \\
\text { tontura persistente } \\
\text { postural paroxística } \\
\text { tratados com } \\
\text { sertralina. }\end{array}$ & $\begin{array}{l}\text { Metodologia: Os } 91 \text { pacientes a receberem tratamento com } \\
\text { 50-200 mg/dia de Sertralina foram divididos em dois grupos: } 46 \text { pacientes } \\
\text { receberam TCC adicional; } 45 \text { pacientes não receberam TCC adicional. } \\
\text { Todos os pacientes foram submetidos aos questionários DHI; HDRS e } \\
\text { HARS. } \\
\text { Resultados: Após } 8 \text { semanas os scores dos questionários DHI, HARS } \\
\text { e HDRS foram substancialmente inferiores em ambos os grupos, mas } \\
\text { especialmente no grupo de pacientes submetidos a TCC adicional, nas } \\
\text { semanas } 4 \text { e } 8 \text { ( } p \text { < 0,005). } \\
\text { A dose de sertralina foi substancialmente inferior no grupo de pacientes } \\
\text { submetidos a TCC adicional e com menos efeitos laterais. } \\
\text { Limitações: Follow-up de apenas } 8 \text { semanas. }\end{array}$ & B & 2 \\
\hline
\end{tabular}

N: tamanho da amostra; M: masculino, F: feminino, GR: grau de recomendação, NE: nível de evidência

\section{DISCUSSÃO}

O benefício do uso da TCC como terapia adjuvante à reabilitação vestibular, terapêutica farmacológica ou outras abordagens multidisciplinares é consensual entre os estudos. São reportados um melhor controlo postural e da tontura. ${ }^{1,2,4}$ São ainda referidas reduções mais significativas dos níveis de ansiedade e sintomas depressivos nestes doentes, e redução das doses de medicação necessárias para o tratamento. ${ }^{1-4}$

Trinidade et a ${ }^{1}$ consideram que à semelhança de outras doenças funcionais e psiquiátricas que causam vertigem, a TPPP deve ser tratada com um plano multidisciplinar, que inclui terapêutica farmacológica, educação e informação do doente, reabilitação vestibular e TCC. Apresentam um artigo de revisão sistemática, que inclui 15 artigos, com data de publicação posterior a 2005, dois deles usando apenas o resumo. Inclui quatro artigos que abordam especificamente o benefício da TCC na abordagem da TPPP.

Os autores traçam considerações relativas aos benefícios da TCC nestes doentes, nomeadamente num mais eficaz controlo sintomático e necessidade de doses me- nores de medicação. Faz, no entanto, menção à ausência de estudos com seguimento superior a um ano.

Por outro lado, Popkirov et al ${ }^{2}$ apresentam um artigo de revisão não sistemática que aborda a fisiopatologia da TPPP e estratégias de gestão. No que toca à abordagem da TPPP usando a TCC, faz referência a 11 artigos bibliográficos, que datam desde 1994 a 2018.

Analisa vários programas de TCC e sumariza os seus princípios comuns: a) educação do doente, b) técnicas de auto-observação, c) identificação e controlo de resposta emocional à vertigem/ tontura, d) controlo de fatores psicossociais, e) adaptação do tratamento para garantir maior funcionalidade no dia-a-dia.

A maioria dos estudos reporta uma melhoria dos sintomas de vertigem e ansiedade, no entanto nem sempre com repercussão na incapacidade, e, até então, sem evidência de extensão dos seus benefícios além de um ano de seguimento.

Conclui que a TCC parece ser uma intervenção útil, preferencialmente combinada com outros métodos de tratamento, mas sem evidência de benefício a longo prazo. 
Um estudo caso-controlo apresentado por Yu et al ${ }^{3} \mathrm{em}$ 2018, que incluiu 91 participantes (46 casos e 45 controlos), procura estudar o papel da TCC como potenciador do tratamento com sertralina em doentes com TPPP.

Os 91 doentes, sob tratamento com 50-200 mg/dia de sertralina, foram divididos em dois grupos: 46 receberam TCC adicional; 45 não receberam TCC adicional. Todos os doentes foram submetidos aos questionários Dizziness Handicap Inventory (DHI); Hamilton Depression Rating Scale (HDRS) e Hamilton Anxiety Rating Scale (HARS). O estudo teve um seguimento de apenas 8 semanas.

Após 8 semanas os scores dos questionários DHI, HARS e HDRS foram substancialmente inferiores em ambos os grupos, mas especialmente no grupo de doentes submetidos a TCC adicional, nas semanas 4 e $8(p<0,005)$.

De referir ainda que a dose de sertralina foi substancialmente inferior no grupo de doentes submetidos a TCC adicional, que reportaram, da mesma forma, menos efeitos secundários.

Schmid et al ${ }^{4}$ apresentam também os resultados de um estudo de caso controlo de 2018, que tem como objetivo determinar a eficácia de um programa combinado de TCC, reabilitação vestibular e psico-educação em doentes com e sem patologia vestibular. Tem uma amostra de 32 participantes, que foi subdividida em dois grupos: 16 casos, doentes com tontura com patologia vestibular, e 16 controlos, doentes com tontura e sem patologia vestibular. Ambos os grupos foram submetidos ao mesmo programa terapêutico e todos os doentes foram submetidos a questionários (Brief Symptom Inventory - BSI e DHI) pré e pós-tratamento.

Foram destacados os seguintes resultados:

- O equilíbrio postural melhorou significativamente no grupo de doentes com patologia vestibular ( $p=0,003$ ), mas tal não se verificou no grupo controlo;

- Os índices dos questionários DHI e BSI melhoraram significativamente no grupo sem patologia vestibular, no entanto, não houve qualquer impacto nestes índices no grupo de doentes com patologia vestibular;

- Os índices de ansiedade fóbica melhoraram substancialmente para ambos os grupos, com maior impacto no grupo sem patologia vestibular.

Em suma, a combinação de TCC, reabilitação vestibular e educação do doente provou melhorar os índices psicológicos no grupo sem patologia vestibular, no entanto, o impacto não foi tão substancial no grupo com patologia vestibular, apesar da melhoria marcada no equilíbrio postural em ambos.
Da análise do artigo destacam-se duas grandes limitações: a heterogeneidade dos grupos caso e controlo, com substancial diferença de idades (sendo que no grupo dos casos os participantes têm uma idade média de 60 anos, e no grupo dos controlos de 45 anos), e o muito breve período de seguimento, de apenas 2 meses.

A nossa pesquisa bibliográfica reforça ainda o papel dos sintomas ansiosos e psicológicos no ciclo de maladaptação da TPPP, reforçando a importância da TCC como parte integrante do tratamento destes doentes. ${ }^{5}$

Whalley e Cane $^{5}$ publicaram em 2017 um trabalho propondo um modelo cognitivo-comportamental para a TPPP. Falam sobre a evolução do seu diagnóstico e tratamento, elucidando o papel da TCC nestes doentes. Os autores consideram que na TPPP, os doentes que apresentam comportamentos de evasão e preventivos, assim como uma maior suscetibilidade a desenvolverem sintomatologia psicossomática determinada pelo medo da tontura, apresentam um certo grau de disfunção cognitivo-comportamental. Nestes doentes, a vertigem tende a adquirir um caráter persistente. Consideram, portanto, a TCC como parte essencial da metodologia do tratamento da TPPP.

Apesar dos resultados dos estudos serem bastante consensuais nas suas conclusões, destaca-se o facto de apenas cinco artigos terem sido incluídos nesta revisão. Os trabalhos de revisão identificados na pesquisa baseiam-se em literatura antiga e/ou desatualizada. Da mesma forma, os artigos têm, na sua maioria, amostras pequenas, e períodos de seguimento de 2 semanas a 2 meses, sendo que apenas um se estende a um ano. Os achados reforçam a necessidade de investimento nesta área, nomeadamente estudos mais recentes, com amostras maiores e seguimento mais prolongado.

\section{CONCLUSÃO}

A terapia cognitivo-comportamental parece ser uma opção terapêutica válida, principalmente em associação com outras medidas (farmacológica e/ou reabilitação vestibular e/ou educação do doente). A redução na dose de sertralina no grupo de doentes que foram submetidos a terapia cognitivo-comportamental durante as 8 semanas de seguimento verificados por Yu et al, reforça a importância do desenvolvimento de estudos nos mesmos moldes, com períodos de seguimento mais alargados, com resultados bastante mais conclusivos no que toca à recomendação desta associação terapêutica.

Num país com uma realidade como a portuguesa, onde apenas uma pequena fração dos doentes com vertigem 
têm acesso a terapia dirigida, como a reabilitação vestibular, os restantes recursos ao dispor adquirem um papel primordial. Em doentes com uma fraca resposta à terapêutica convencional medicamentosa, a TCC poderá representar uma solução viável, e ter um impacto significativo no que toca ao prognóstico e qualidade de vida destes doentes.

Apesar das conclusões deste artigo indicarem um óbvio benefício no uso da TCC, estudos adicionais são imperativos, com amostras maiores e períodos de seguimento mais longos, de forma a determinar mais assertivamente o papel desta terapia no curso da doença.

CONFLITOS DE INTERESSE: Os autores declaram não ter qualquer conflito de interesse na realização do presente trabalho.

FONTES DE FINANCIAMENTO: Não houve qualquer fonte de financiamento na realização do presente trabalho.

PROVENIÊNCIA E REVISÃO POR PARES: Não comissionado; revisão externa por pares.

CONFLICTS OF INTEREST: The authors declare that they have no conflicts of interest.

FINANCIAL SUPPORT: This work has not received any contribution, grant or scholarship.

PROVENANCE AND PEER REVIEW: Not commissioned; externally peer reviewed.

\section{REFERÊNCIAS}

1. Trinidade A, Goebel JA. Persistent postural-perceptual dizziness-a systematic review of the literature for the balance specialist. Otol Neurotol. 2018;39:1291-303. doi: 10.1097/ MAO.0000000000002010.

2. Popkirov S, Stone J, Holle-Lee D. Treatment of Persistent Postural-Perceptual Dizziness (PPPD) and Related Disorders. Curr Treat Options Neurol. 2018;20:50. doi: 10.1007/ s11940-018-0535-0.

3. Yu YC, Xue H, Zhang YX, Zhou J. Cognitive behavior therapy as augmentation for sertraline in treating patients with persistent postural-perceptual dizziness. Biomed Res Int. 2018;2018:8518631. doi: 10.1155/2018/8518631.

4. Schmid DA, Allum JH, Sleptsova M, Gross S, Gaab J, Welge-Lüssen A,et al. Effects of a program of cognitive-behavioural group therapy, vestibular rehabilitation, and psychoeducational explanations on patients with dizziness and no quantified balance deficit, compared to patients with dizziness and a quantified balance deficit. J Psychosom Res. 2018;105:21-30. doi:10.1016/j.jpsychores.2017.11.020.

5. Whalley MG, Cane DA. A cognitive-behavioral model of persistent postural-perceptual dizziness. Cogn Behav Pract. 2017;24: 72-89. doi:10.1016/j.cbpra.2016.03.003. 